\title{
Quantum-well-induced ferromagnetism in thin films
}

Niklasson, A.M.N.; Mirbt, S.; Skriver, Hans Lomholt; Johansson, B.

\section{Published in:}

Physical Review B

Link to article, DOI:

10.1103/PhysRevB.56.3276

Publication date:

1997

\section{Document Version}

Publisher's PDF, also known as Version of record

Link back to DTU Orbit

Citation $(A P A)$ :

Niklasson, A. M. N., Mirbt, S., Skriver, H. L., \& Johansson, B. (1997). Quantum-well-induced ferromagnetism in thin films. Physical Review B, 56(6), 3276-3280. https://doi.org/10.1103/PhysRevB.56.3276

\section{General rights}

Copyright and moral rights for the publications made accessible in the public portal are retained by the authors and/or other copyright owners and it is a condition of accessing publications that users recognise and abide by the legal requirements associated with these rights.

- Users may download and print one copy of any publication from the public portal for the purpose of private study or research.

- You may not further distribute the material or use it for any profit-making activity or commercial gain

- You may freely distribute the URL identifying the publication in the public portal

If you believe that this document breaches copyright please contact us providing details, and we will remove access to the work immediately and investigate your claim. 


\title{
Quantum-well-induced ferromagnetism in thin films
}

\author{
A. M. N. Niklasson and S. Mirbt \\ Condensed Matter Theory Group, Physics Department, Uppsala University, S-75121 Uppsala, Sweden
}

H. L. Skriver

Center for Atomic-scale Materials Physics and Department of Physics, Technical University of Denmark, DK-2800 Lyngby, Denmark

\author{
B. Johansson \\ Condensed Matter Theory Group, Physics Department, Uppsala University, S-75121 Uppsala, Sweden
}

(Received 28 February 1997)

\begin{abstract}
We have used a first-principles Green's-function technique to investigate the magnetic properties of thin films of Rh, Pd, and Pt deposited on a fcc Ag (001) substrate. We find that the magnetic moment of the film is periodically suppressed and enhanced as a function of film thickness. The phenomenon is explained in terms of quantum-well states moving through the Fermi level with increasing film thickness.
\end{abstract}

[S0163-1829(97)07930-7]

\section{INTRODUCTION}

In recent years there have been a number of predictions of magnetism in monolayers of the late $4 d$ and $5 d$ transition metals deposited on Ag (001) substrates. ${ }^{1-7}$ One argument for the magnetic transition is based on the energy band narrowing caused by the reduction in coordination number when going from a three-dimensional bulk to a twodimensional film. In a Stoner picture the corresponding increase in the state density at the Fermi level may lead to onset of a magnetism. An alternative explanation, which will be discussed in the present work, is based on the quantumwell $(\mathrm{QW})$ states $^{8-11}$ that are formed in the overlayer film and that moves through the Fermi level as the film thickness increases whereby the state density at the Fermi level is periodically enhanced and suppressed. The QW concept is particularly fruitful when considering possible magnetism in overlayer films consisting of more than a single monolayer.

Recently Mirbt et al. ${ }^{12}$ showed by means of firstprinciples calculations that QW states quench the magnetization in thin films of Pd on a Ag (001) substrate. Surprisingly, the quenching occurs only for certain film thicknesses, despite the fact that bulk Pd is magnetic at the lattice spacing of Ag. The QW states are also found to give a small constant shift of the magnetization profile in $\mathrm{Fe} / \mathrm{Cu} / \mathrm{Fe}$ trilayers. ${ }^{13}$ Indication of such a shift has in fact been observed in surface sensitive second-harmonic-generation measurements of the induced magnetization in thin films of $\mathrm{Cu}$ deposited on a $\mathrm{Co}$ fcc (001) substrate. ${ }^{14}$ Here we explain how the QW mechanism may lead to a magnetic transition in thin films of Rh, Pd, and Pt deposited on a Ag (001) substrate.

The simple Stoner criteria for magnetization is not exactly correct for our layered systems where the perpendicular symmetry is broken and each single monolayer can behave differently but still not completely independent, especially since the QW states are extended over the entire overlayer films. However, we will still analyze the tendency towards magnetization in terms of the Stoner picture, but only qualitatively, i.e., that an increased state density at the Fermi level may lead to onset of magnetization. This qualitative use of the Stoner criteria is also confirmed by our calculations.

The paper is organized as follows. In Sec. II we outline the first-principles Green's-function technique used in the calculations. In Sec. III we present the magnetization effects in the overlayer films and explain how the spin polarization which appears for certain film thicknesses may be understood in terms of QW states. In particular, we analyze the QW dispersion and show how it is related to the Fermi surface and the total states density.

\section{CALCUlational DETAILS}

The Green's-function technique used in the present work is implemented by Skriver and Rosengaard ${ }^{15}$ and has been applied in several studies of surface and interface magnetism. ${ }^{16,17,13}$ It is based on the linear muffin-tin orbitals method $^{18,19}$ in the tight-binding, ${ }^{20}$ frozen core, and atomicsphere approximations in conjunction with the local-spindensity approximation as parametrized by Vosko, Wilk, and Nusair. ${ }^{21}$ The Green's-function technique does not rely on a slab or supercell geometry and therefore ensures a correct description of the loss of translational symmetry perpendicular to the interface. Furthermore, the application of the principle layer technique ${ }^{22}$ leads to a minimal computational effort which scales linearly with the number of atomic layers.

The properties of the QW states are most conveniently discussed in terms of the spectral density function

$$
D^{\sigma}\left(\mathbf{k}_{\|}, E\right)=\frac{1}{\pi} \operatorname{Im} \operatorname{Tr} G^{\sigma}\left(\mathbf{k}_{\|}, E\right),
$$

which we calculate from the Green's function, $G^{\sigma}\left(\mathbf{k}_{\|}, E\right)$ of spin $\sigma$, wave vector $\mathbf{k}_{\|}$, and real energy $E$, by means of an analytical continuation from a linear mesh below the real axis in the complex energy plane. To obtain a layer resolved spectral density the trace over each individual monolayer is calculated separately. The spectral density may be seen as 


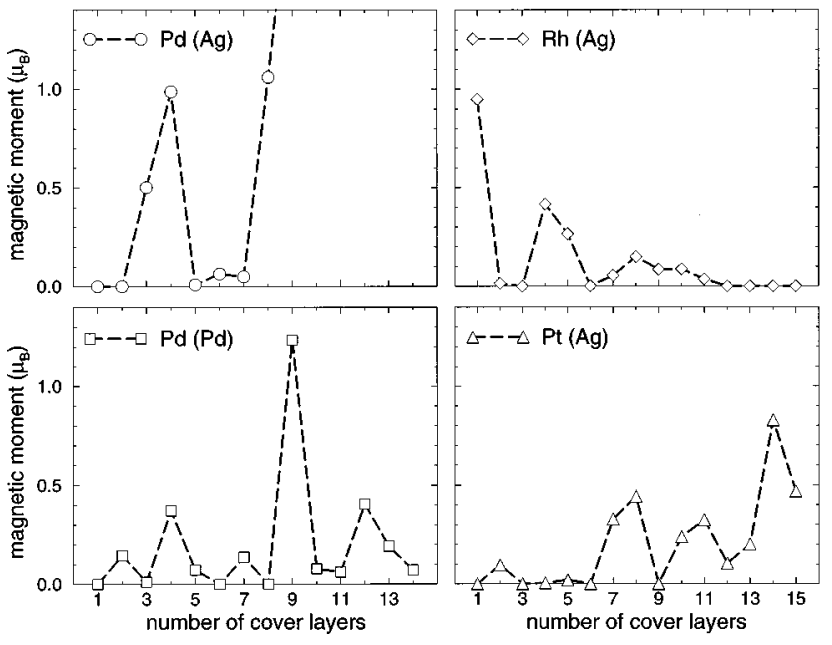

FIG. 1. The total magnetization, summed over all cover layers, in thin films on a $\mathrm{Ag}(001)$ substrate, as a function of film thickness. The lattice constants are those of the atomic label given in the parenthesis.

the $k$-resolved state density, which integrated over the twodimensional Brillouin zone (2D-BZ) gives the total state density.

\section{QUANTUM-WELL-INDUCED MAGNETIZATION}

In Fig. 1 we display the calculated total magnetization, i.e., the magnetic moment of the entire film, of thin films of $\mathrm{Pd}, \mathrm{Rh}$, and Pt deposited on a Ag (001) substrate. Surprisingly, the magnetization exhibits a highly irregular behavior as a function of film thickness. For instance, in the case of $\mathrm{Rh}$, which has a large magnetic moment for a single monolayer, the magnetization disappears for 2 and $3 \mathrm{ML}$ but reappears for 4 and 5 ML. Eventually the magnetization decays to zero as the film grows beyond $10 \mathrm{ML}$. A similar behavior is found for $\mathrm{Pt}$ and for $\mathrm{Pd}$ at the Pd lattice spacing while $\mathrm{Pd}$ at the Ag lattice spacing as discussed by Mirbt et $a l .{ }^{12}$ remains magnetic in the thick-film limit. The stability of the magnetic configurations was investigated by comparing the total energies of the magnetic and the nonmagnetic solution. All the magnetic configurations where found to be stable.

Mirbt et al. showed how the formation of QW states may quench the magnetization in thin films of $\mathrm{Pd}$ on top of $\mathrm{Ag}$ (001). ${ }^{12}$ In the following we show in more detail how the presence of QW states may also induce magnetism in films of $\mathrm{Rh}, \mathrm{Pd}$, and $\mathrm{Pt}$.

\section{A. Formation of QW states}

The formation of quantum wells in thin films of metal deposited on a Ag substrate may be explained in terms of the bulk band structures shown in Fig. 2. Of particular interest are electrons in the $\Gamma_{25^{\prime}}-\Delta_{5}-X_{5}$ band of the late transition metal which cannot scatter into the Ag substrate because there are no available states of $\Delta_{5}$ symmetry. As a result, these electrons experience total reflection at the metal-metal interface, and since they are also reflected at the surface, they are effectively confined to the overlayer film.

The deposition of a thin film on a Ag substrate changes the periodic Born-Karman boundary conditions to those of total reflection at the film boundaries leading to the formation of QW states. The corresponding quantization may be described in terms of the phase accumulation picture, ${ }^{23,24}$ which expresses the condition for constructive interference of a QW state of wave vector $k_{n}^{\perp}$ as

$$
2 k_{n}^{\perp} D+\varphi_{L}+\varphi_{R}=2 \pi n,
$$

where $D$ is the film thickness, $\varphi_{L(R)}$ is the scattering phase shift at the left (right) interface, and $n$ is an integer quantum number. If we measure $k^{\perp}$ in units of $2 \pi / a$ where $a$ is the fcc lattice constant, assume $D=N a / 2$ where $N$ is the layer thickness in units of monolayers, and define $\varphi=\left(\varphi_{L}+\varphi_{R}\right) / 2 \pi$ we have that

$$
k_{n}^{\perp}=(n+\varphi) / N .
$$
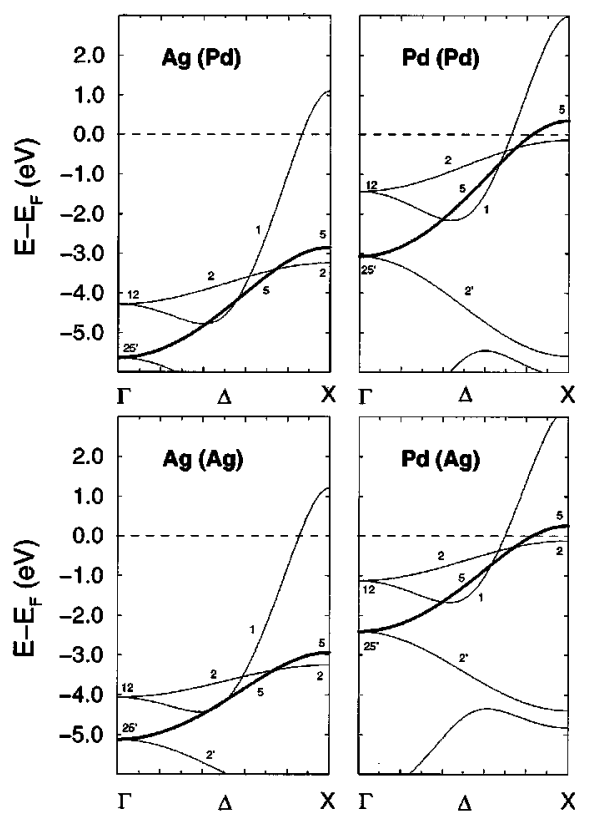
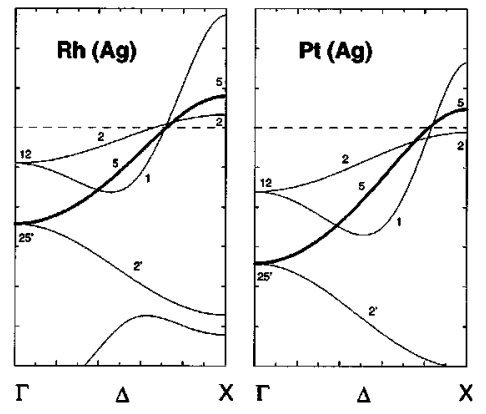

FIG. 2. The bulk bands of the Ag (001) substrate compared with the bulk bands of the cover material. The lattice constants are those of the atomic label given in the parenthesis. The $\Gamma_{25^{\prime}}$ $\Delta_{5}-X_{5}$ bands are indicated by fat lines. Electrons with this symmetry cannot scatter into the $\mathrm{Ag}$ substrate because there are no available states of $\Delta_{5}$ symmetry around the Fermi level in Ag. These bands form part of the Fermi surface with the nesting features marked by arrows in Fig. 4 . 
This quantum condition combined with periodic boundary conditions in the two dimensions parallel to the film lead to a quantization of the bulk bands of the film material which in the limit of thick films is equivalent to the usual Born-von Karman quantization. The quantization may be understood in the following way: Deep inside a thick film the wave functions must fulfill the periodic boundary conditions of the bulk material and at the interfaces they are subjected to total reflection. Only wave functions which satisfy both conditions may exist. In the limit of thin films such a quantization of the bulk bands is only approximately valid, but as we show below the periodic behavior of the QW states as well as their dispersion may qualitatively be described in terms of such a simple quantum condition.

\section{B. Periodicity of the QW states}

According to the quantum condition (3) the energy levels of a film quantum well may be obtained from the bulk band structure $\varepsilon$ as

$$
\varepsilon_{\mathrm{qw}}=\varepsilon\left(k_{n}^{\perp}, \mathbf{k}^{\|}\right) .
$$

It follows that QW states in the bands which contribute to the Fermi surface will periodically cross the Fermi level as the film thickness increases. There are in fact two different types of periods. The first period $P_{c}$ is given by the crossing of the QW states in the limit where the width of the QW changes continuously (c). The second period $P_{d}$ stems from the discreteness $(d)$ of the overlayer lattice and occurs when $k_{F}^{\perp}>1 / 2$. In this case the wavelength $1 / k_{F}^{\perp}$ is less than $2 \mathrm{ML}$, and due to the discrete sampling of the QW energies at each integer number of overlayers, the QW branches appear to cross the Fermi level with a different period and in the opposite direction of the continuous case. This discrete sampling phenomenon is usually referred to as the aliasing effect.

The period $P_{c}$ may be obtained in units of monolayers (ML) from the quantization condition (3) by calculating the spacer thickness difference, $N_{2}-N_{1}$, between two adjacent QW states, $n_{1}$ and $n_{2}$, at the Fermi level, i.e.,

$$
P_{c}=N_{2}-N_{1}=\left(n_{2}-n_{1}\right) / k_{F}^{\perp}=1 / k_{F}^{\perp} .
$$

The second period $P_{d}$ is obtained by replacing the quantum number $n$ by $m=N-n$ which gives

$$
P_{d}=N_{2}-N_{1}=\left(m_{2}-m_{1}\right) /\left(1-k_{F}^{\perp}\right)=1 /\left(1-k_{F}^{\perp}\right),
$$

which only differs from Eq. (5) by the subtraction of a reciprocal-lattice vector in the denominator.

\section{QW-induced magnetization}

The QW states which periodically cross the Fermi level as the thickness of the overlayer film changes, will lead to oscillations in the state density at the Fermi level as well as in the magnetic susceptibility around its bulk value, with periods given by the Fermi wave vectors. If we now deposit overlayers of metals which are on the border of being ferromagnetic the overlayer may oscillate between a magnetic and a nonmagnetic state. Such an oscillatory magnetic behavior may be viewed as a direct consequence of the $\mathrm{QW}$ states formed in the overlayer.
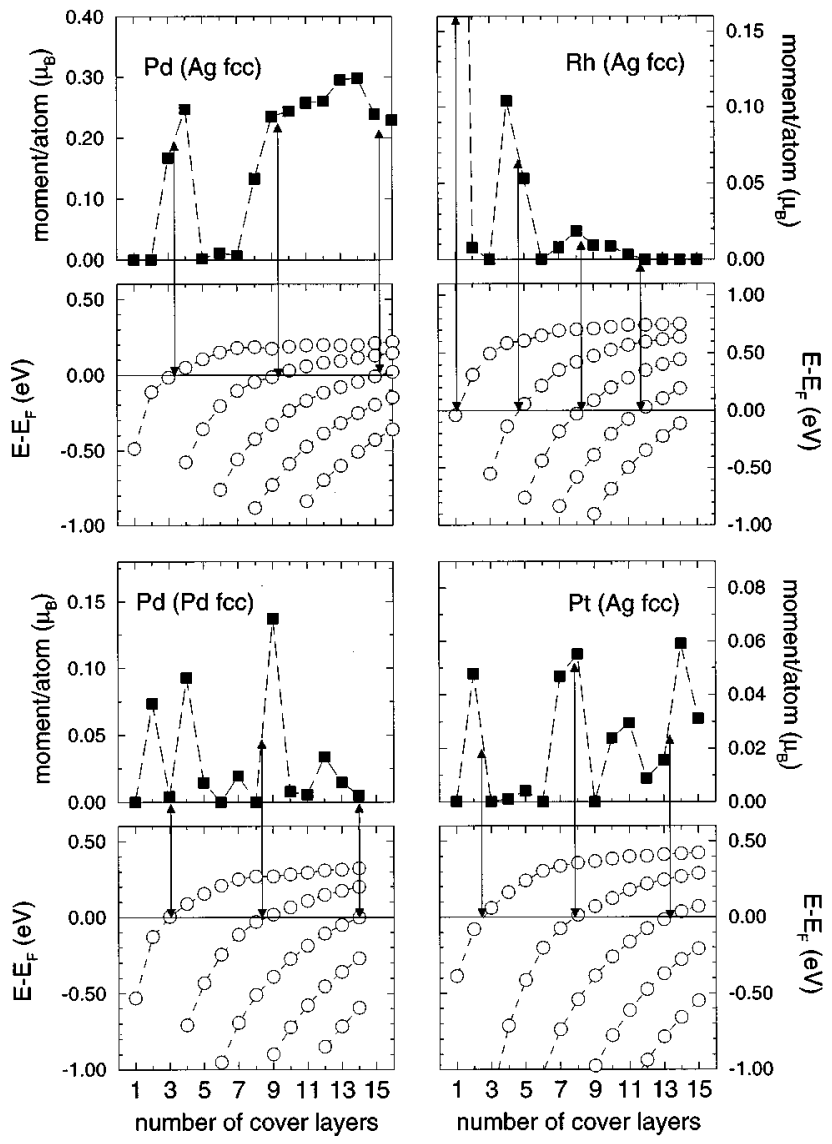

FIG. 3. Comparison between the average magnetization per atom and the periodic behavior of the QW states corresponding to the quantization of the $\Delta_{5}$-symmetry bands near the Fermi level at the $\bar{\Gamma}$ point. The average magnetization is simply the total magnetization shown in Fig. 1 divided by the number of covering layers. The arrows are guides to the eyes when comparing between the Fermi-level crossing of QW branches and the magnetization.

In Fig. 3 we make a comparison between the periodic crossings of the QW states corresponding to the quantization of the $\Delta_{5}$-symmetry bands at $\bar{\Gamma}$ in the two-dimensional Brillouin zone (2D-BZ) and the average magnetization per atom in the overlayer films. In the case of $\mathrm{Rh}$ we find that the magnetization for 1,4 , and 7 ML coincides with a QW state near the Fermi level. The magnetization for a single monolayer $\mathrm{Rh}$ has earlier been considered to be an example of a magnetization which occurs due to the band narrowing caused by the reduction in coordination number at the surface. ${ }^{4}$ Here we complement this picture by the QW mechanism and point out that if there is no QW state near the Fermi level the reduced coordination may not be sufficient to induce magnetism. This is clearly the case for $\mathrm{Pd}$ and $\mathrm{Pt}$ where a single overlayer is nonmagnetic.

We have not mentioned the QW states corresponding to the quantization of the $\Delta_{2}$-symmetry bands. For Rh these states do in fact cross the Fermi level at $\Gamma$, but due to their dispersion in the 2D-BZ they turn out to have less influence on the total state density and the magnetization.

As seen in Fig. 3 there is good overall agreement between the magnetization of the overlayer film and the periodic crossing of QW branches at the Fermi level. However, the agreement is not perfect, and for example, $3 \mathrm{ML}$ of Pd in the 

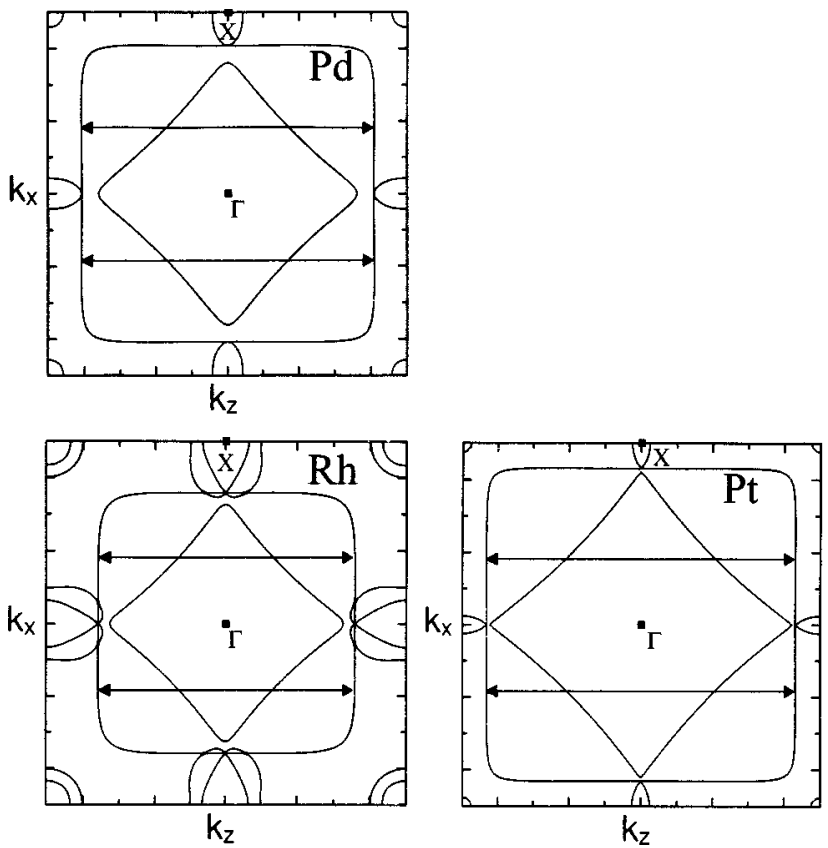

FIG. 4. The Fermi surfaces of bulk cover materials of Pd, Rh, and Pt. The nesting features, i.e., the large flat parts with no dispersion, are indicated by arrows. Along $\Gamma-\mathrm{X}$ the nesting sheets are given by the $\Delta_{5}$-symmetry bands crossing the Fermi level.

lattice constant of Pd is not magnetic even if there is a crossing of a QW branch at $\bar{\Gamma}$ for this thickness. The reason for this discrepancy is simply that here we have only considered QW states at $\bar{\Gamma}$. To understand the total state density we need to analyze the QW dispersion and contributions from the entire 2D-BZ.

The periods of the QW states correspond almost exactly to the periods derived from Eq. (6) with the Fermi wave vectors extracted from the bulk band calculations displayed in Fig. 2. The period $P_{d}$ calculated from the bulk properties, 5.7 ML for Pd and Pt and 3.5 ML for Rh, agrees very well with the distance between the last two intersections of the QW branches and the Fermi level, where the films are most bulklike.

\section{The Fermi surface and the dispersion of the QW states}

So far we have only discussed the influence of QW states at $\bar{\Gamma}$. However, QW states from the entire 2D-BZ will of course contribute to the total state density at the Fermi level. To obtain a correct picture of the influence of the QW states we must therefore consider their dispersion. Of major importance is how the nesting features of the Fermi surface, i.e., the large flat parallel sheets of the Fermi surfaces of Pd, Pt, and $\mathrm{Rh}$, as indicated by the arrows in Fig. 4, will lead to a very weak dispersion of the QW states and thereby further increase the changes in the total state density near the Fermi level. Along $\Gamma-X$ these flat Fermi sheets correspond to the $\Delta_{5}$-symmetry bands crossing the Fermi level.

One may obtain a simple understanding of the dispersion of the QW states by a linearization of the quantized bulk bands (4) around the Fermi wave vector $k_{F}^{\perp}\left(\mathbf{k}^{\|}\right)$. One finds
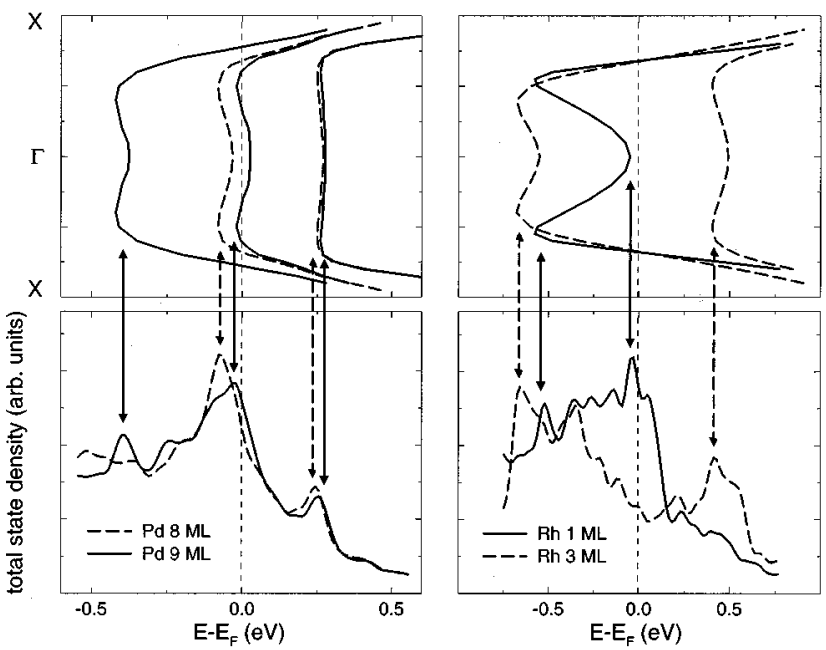

FIG. 5. A comparison between the dispersions of the QW states in the 2D-BZ, corresponding to the flat nesting parts of the bulk Fermi surfaces, and the van Hove peaks in the total state density. The two upper panels show the dispersion of QW states in the 2D-BZ for Pd films with 8 and $9 \mathrm{ML}$ and Rh films with 1 and 3 ML. The two lower panels show the total state density. As implied by the the linearization in Eq. (7) the QW dispersions resemble the flat nesting features of the Fermi surfaces. This leads to the pronounced van Hove singularities in the total state density as indicated by arrows.

$$
\varepsilon_{q w}\left(k_{n}^{\perp}\right)-\varepsilon_{F},\left.\approx\left[k_{\mathrm{n}}^{\perp}-k_{F}^{\perp}\left(\mathbf{k}_{\|}\right)\right] \frac{\partial \varepsilon_{\mathrm{qw}}}{\partial k^{\perp}}\right|_{k^{\perp}=k_{F}^{\perp}},
$$

which gives a direct connection between the dispersion of the QW states and the Fermi surface of the bulk material. It follows, that if the band velocity $\left.\left(\partial \varepsilon_{\mathrm{qw}} / \partial k^{\perp}\right)\right|_{k^{\perp}=k_{F}^{\perp}}$ and the scattering phase shift $\varphi$, which may depend on $\mathbf{k}^{\|}$, does not vary too rapidly the energy dispersion of a particular QW state close to the Fermi level should have the shape of the Fermi surface $k_{F}^{\perp}\left(\mathbf{k}^{\|}\right)$. In the case of $\mathrm{Rh}, \mathrm{Pd}$, and $\mathrm{Pt}$, where the Fermi surfaces have large flat parts, i.e., $k_{F}^{\perp}\left(\mathbf{k}_{\|}\right)$is almost independent of $\mathbf{k}_{\|}$as is seen in Fig. 4, the dispersion of the QW states will be very weak. Hence, the total state density, i.e., the spectral density integrated over the 2D-BZ, will be greatly enhanced in a narrow energy region around a particular QW state close to the Fermi level. Within a Stoner picture this may induce the onset of magnetism.

In Fig. 5 we show a few examples of the QW dispersion branches corresponding to the nesting features of the Fermi surface together with the total state density for the cover films. The QW branches clearly have the shape of the nesting parts of the Fermi surfaces as implied by the linearization in Eq. (7). We also observe prominent contributions to the total state density due to the weak QW dispersion and in particular we find pronounced van Hove singularities corresponding to the extremal points of the QW branches. Due to the fourfold symmetry there are four extremal points outside $\bar{\Gamma}$. These extrema will therefore have a stronger impact on the total state density than the $\bar{\Gamma}$ extremum, even if their curvature is somewhat smaller. It is therefore essentially the periodic Fermi level crossing of QW branches at the extrema outside $\Gamma$ which will govern the periodic magnetization. As 
a result the state density at the Fermi level and the magnetic susceptibility will oscillate as a function of the spacer thickness explaining the oscillatory magnetic behavior of the overlayer films. Of course, all states, not only QW states corresponding to the flat parts of the Fermi surface, will contribute to the total state density, but the QW branches with a weak dispersion will have the major impact on the oscillations in the total state density.

An interesting property is found if we investigate the size of the dispersion of the QW branches in Fig. 5. The dispersion decays as a function of film thickness and the curvature at the extremal points of the QW branches decreases. This means an increased band mass as a function of film thickness, especially at the extremal points of the QW branches, which may be important for the electric conductivity and the magnetic interlayer coupling. ${ }^{25}$

\section{SUMMARY AND CONCLUSIONS}

We have shown that thin films of Rh, Pd, and Pt deposit on a $\mathrm{Ag}(001)$ substrate may exhibit an irregular magnetic behavior as a function of film thickness. The phenomenon is interpreted in terms of a QW mechanism which through the nesting properties of the Fermi surfaces of these metals leads to a weak dispersion of the QW states and thereby to large oscillations in the state density close to the Fermi level. One signature of the QW mechanism is the existence of magnetism in a film of an otherwise nonmagnetic metal. Such a magnetic overlayer might just be observed experimentally.

Since other types of layered systems may also exhibit the QW-induced magnetism described in the present paper we mention here the main conditions for this effect. (i) The metal in the overlayer must be close to the transition to a magnetic state, (ii) it must form quantum-well states in a range around the Fermi level on a suitable substrate, and (iii) its Fermi surface should exhibit nesting, i.e., have parallel and flat sheets perpendicular to the plane of the overlayer film. If these conditions are satisfied the system will have large oscillations in the state density at the Fermi level and may exhibit transitions between magnetic and nonmagnetic states as a function of the film thickness.

Several experimental difficulties may prevent the observation of the effect predicted in the present paper. Small changes at the interfaces such as intermixing and vacancies may reduce the effects of the QW states. ${ }^{7}$ Lattice relaxation will probably be of less importance. Relaxation may change the width of the QW but that will only change the phase of the oscillation. Temperature and impurities will also smear out the effect of the QW states. Despite the smearing effects, oscillations in the state density at the Fermi level will remain even if the amplitude may decrease and for example the magnetic susceptibility will still oscillate as a function of film thickness.

\section{ACKNOWLEDGMENTS}

We wish to thank the Swedish Natural Science Research Council (NFR) and the Swedish Materials Consortium No. 9 financed by NUTEK and NFR for valuable support. Center for Atomic-scale Materials Physics is sponsored by the Danish National Research Foundation.
${ }^{1}$ J. Mathon, Rep. Prog. Phys. 51, 1 (1988).

${ }^{2}$ R. L. Fink, C. A. Ballentine, J. L. Erskine, and J. A. ArayaPochet, Phys. Rev. B 41, 10175 (1990).

${ }^{3}$ M. J. Zhu, D. M. Bylander, and L. Kleinman, Phys. Rev. B 43, 4007 (1991).

${ }^{4}$ O. Eriksson, R. C. Albers, and A. M. Boring, Phys. Rev. Lett. 66, 1350 (1991).

${ }^{5}$ S. Blügel, Phys. Rev. Lett. 68, 851 (1992).

${ }^{6}$ B. Ujfalussy, L. Szunyogh, and P. Weinberger, Phys. Rev. B 51, 12836 (1995).

${ }^{7}$ I. Turek, J. Kudronovský , M. Šob, V. Drchal, and P. Weinberger, Phys. Rev. Lett. 74, 2551 (1995).

${ }^{8}$ S. Å. Lindgren and L. Walldén, Phys. Rev. Lett. 59, 3003 (1987).

${ }^{9}$ J. E. Ortega and F. J. Himpsel, Phys. Rev. Lett. 69, 844 (1992).

${ }^{10}$ K. Garrison, Y. Chang, and P. D. Johnson, Phys. Rev. Lett. 71, 2801 (1993).

${ }^{11}$ C. Carbone, E. Vescovo, O. Rader, W. Gudat, and W. Eberhardt, Phys. Rev. Lett. 71, 2805 (1993).

${ }^{12}$ S. Mirbt, B. Johansson, and H. L. Skriver, Phys. Rev. B 53, R13 310 (1996).

${ }^{13}$ A. M. N. Niklasson, S. Mirbt, H. L. Skriver, and B. Johansson, Phys. Rev. B 53, 8509 (1996).
${ }^{14}$ H. A. Wierenga, W. de Jong, M. W. J. Prins, Th. Rasing, R. Vollmer, A. Kirilyuk, H. Schwabe, and J. Kirschner, Phys. Rev. Lett. 74, 1462 (1995).

${ }^{15}$ H. L. Skriver and N. M. Rosengaard, Phys. Rev. B 43, 9538 (1991).

${ }^{16}$ M. Aldén, H. L. Skriver, S. Mirbt, and B. Johansson, Phys. Rev. Lett. 69, 2296 (1992).

${ }^{17}$ S. Mirbt, O. Eriksson, B. Johansson, and H. L. Skriver, Phys. Rev. B 52, 15070 (1995).

${ }^{18}$ O. K. Andersen, Phys. Rev. B 12, 3060 (1975).

${ }^{19}$ H. L. Skriver, The LMTO Method (Springer-Verlag, Berlin, 1984).

${ }^{20}$ O. K. Andersen and O. Jepsen, Phys. Rev. Lett. 53, 2571 (1984).

${ }^{21}$ S. H. Vosko, L. Wilk, and M. Nusair, Can. J. Phys. 58, 1200 (1908).

${ }^{22}$ B. Wenzien, J. Kudrnovsky, V. Drchal, and M. Sob, J. Phys., Condens. Matter. 1, 9893 (1989).

${ }^{23}$ P. M. Echenique and J. B. Pendry J. Phys. C 11, 2065 (1978).

${ }^{24}$ N. V. Smith, N. B. Brookes, Y. Chang, and P. D. Johnson, Phys. Rev. B 49, 332 (1994).

${ }^{25}$ P. Bruno and C. Chappert, Phys. Rev. Lett. 67, 1602 (1991). 